\title{
ESTRUTURA E PADRÃO ESPACIAL DAS POPULAÇÕES DE BACTRIS SETOSA MART E B. HATSCHBACHII NOBLICK EX A. HEND (ARECACEAE) EM UM GRADIENTE ALTITUDINAL, UBATUBA (SP)
}

\author{
Evoni Antunes Monteiro' ${ }^{1}$, Simey Thury Vieira Fisch ${ }^{1 \text { e } 2}$
}

Biota Neotropica v5(n2)-http://www.biotaneotropica.org.br/v5n2/pt/abstract?article+BN00505022005

\author{
Recebido em 20/04/04. \\ Revisado em 04/03/05. \\ Publicado em 01/07/2005.
${ }^{1}$ Programa de Pós-Graduação em Ciências Ambientais- PPG-CA
Universidade de Taubaté- Campus de Ciências Agrárias -Estrada Municipal Dr. José Luiz Cembranelli, 5000 Bairro Itaim. CEP: 12081-010, Taubaté, SP- Brasil
e-mail: evonia@uol.com.br
${ }^{2}$ UNITAU-Universidade de Taubaté, Dep. de Biologia- Campus do Bom Conselho- Praça Marcelino Monteiro, 63. \\ CEP: 12030-010, Taubaté, SP- Brasil \\ e-mail: simey@unitau.br
}

\begin{abstract}
A biology study of Bactris setosa Mart and B. hatschbachii Noblick ex A. Hend was made with the aim of obtaining information about structure and spatial pattern of Bactris palm genus in different stretches in atlantic rain forest in Ubatuba-SP, to asses the population of these species in the altitude gradient. Samples were taken in six altitudes (0 m - on sea level, 100, 200, 400, 600 and $850 \mathrm{~m}$ ) in round plots of $100 \mathrm{~m}^{2}$. In these plots, the two species were measured. Populations were divided into five size class: classe 1 (acaulescent); classe 2 (1 a $50 \mathrm{~cm}$ ); classe 3 (51 a $100 \mathrm{~cm})$; classe 4 (101 a $200 \mathrm{~cm})$; classe 5 (> $200 \mathrm{~cm})$. Both populations present a predominance of individual in the class following the seedling class. At sea level both species were abundant (B. hatschbachii, $472 \mathrm{ind} /$ ha and B. setosa, $312 \mathrm{ind} / \mathrm{ha}$ ) and in the slope B. hatschbachii was more numerous in $400 \mathrm{~m}$ (400 ind/ha) of altitude and in $600 \mathrm{~m} \mathrm{~B}$. setosa (148 ind/ha) was more numerous, in $850 \mathrm{~m}$ the two species were not sampled. The results of Morisita index (Id) showed that the spatial pattern for the two species populations was clumped. The populational pattern presented showed that those palm troes are restricted to favorable environment.
\end{abstract}

Key words: spatial distribution, Atlantic Rain Forest, palm ecology.

\section{Resumo}

Foi feito um estudo da estrutura e padrão espacial das palmeiras Bactris setosa Mart. e B. hatschbachii Noblick ex A. Hend., em trechos de mata atlântica no município de Ubatuba-SP, para se avaliar as populações destas espécies no gradiente de altitude. Nestes locais foram realizadas amostragens em seis altitudes (0 m - nível do mar, 100, 200, 400, 600, 850 m) em parcelas circulares de $100 \mathrm{~m}^{2}$, onde todos os indivíduos das duas espécies foram medidos. As populações foram divididas em cinco classes de tamanhos: classe 1 (acaule); classe 2 (1 a $50 \mathrm{~cm}$ ); classe 3 (51 a $100 \mathrm{~cm}$ ); classe 4 (101 a $200 \mathrm{~cm}$ ); classe 5 (> $200 \mathrm{~cm}$ ). Ambas populações apresentaram predominância de indivíduos na classe seguinte a de plântulas. Ao nível do mar, as duas espécies foram abundantes (B. hatschbachii, 472 ind/ha e B. setosa, 312 ind/ha) e na encosta a espécie $B$. hatschbachii foi mais populosa a $400 \mathrm{~m}$ de altitude (400 ind/ha) e B. setosa a $600 \mathrm{~m}$ (148 ind/ha), a $850 \mathrm{~m}$ as duas espécies não foram amostradas. O índice de Morisita (Id) indicou um padrão espacial agregado para as populações de ambas espécies. O padrão populacional apresentado demonstrou que estas palmeiras são restritas a ambientes favoráveis.

Palavras-chave: Distribuição espacial, Floresta Atlântica, Ecologia de Palmeiras.

http://www.biotaneotropica.org.br 


\section{INTRODUÇÃO}

A altitude tem sido considerada como um gradiente complexo de variação atuando em conjunto com a topografia e muitos fatores ambientais. $\mathrm{O}$ aumento da altitude vem sendo estudado e observado como influenciador na diminuição no número de espécies e a redução do tamanho das plantas nas florestas montanas (Richards 1996, Grubb 1977, Gentry 1988, Kent \& Coker 1992). Alguns estudos relacionam a diminuição do número de espécies às freqüentes neblinas, precipitações e as baixas temperaturas nos topos dos morros. As palmeiras em especial, tendem a redução de espécies em altitudes mais elevadas, tornando-se comum a predominância de uma ou poucas espécies evolutivamente mais adaptadas (Grubb 1977, Edward \& Grubb 1982, Ewel \& Bigelow 1996, Lieberman et a.l. 1996, Svenning 2001).

Na floresta ombrófila densa atlântica, conhecida comumente como mata atlântica, destacam-se no subosque florestal as palmeiras dos gêneros Astrocaryum, Bactris e Geonoma. Em função do seu pequeno porte, muitas destas palmeiras acabam não sendo amostradas em levantamentos fitossociológicos (Fisch 1999). Poucas espécies de subosque da mata atlântica foram enfoque de estudos populacionais, destacando-se a acaulescente Attalea humilis (Souza et al. 2000) e, a de floresta semi decídua, Geonoma brevispatha (Souza et al. 2003).

Nas encostas da Serra do Mar, na região de Ubatuba (SP), o gênero Bactris encontra-se representado por duas espécies típicas do subosque florestal: B. hatschbachii Noblick ex A. Hend e B. setosa Mart. As espécies são pouco conhecidas, sendo que a primeira não se encontra amostrada em 24 levantamentos feitos em ambientes florestais no estado de São Paulo (Fisch 1999). Dessa forma, estudos que permitam compreender a distribuição das populações dessas palmeiras, além de as tornarem mais conhecidas, podem ser ferramentas úteis para sua conservação no bioma em que estão inseridas.

O presente estudo busca a compreensão da distribuição das populações de Bactris hatschbachii e $B$. setosa, procurando verificar as alterações ocasionadas pela altitude na estrutura e no padrão espacial destas palmeiras.

\section{MATERIAL E MÉTODOS}

\section{Características das áreas de estudo e das espécies}

O estudo foi desenvolvido em uma área de restinga e em um trecho de encosta na Serra do Mar- Núcleo Picinguaba, Ubatuba, SP (figura 1). A área de restinga situa-se nas

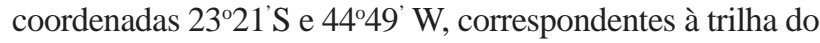

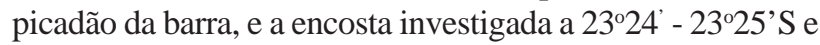
$45^{\circ} 08^{\prime}-45^{\circ} 08^{\prime} \mathrm{W}$, trilha da vargem grande. O clima dessa região é tropical chuvoso com temperaturas altas, e muito úmido no

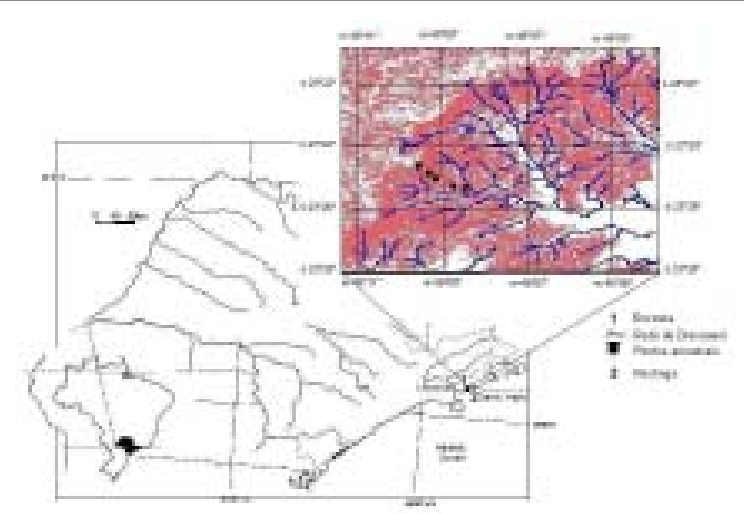

Figura 1: Mapa da localização geográfica das áreas amostradas.

verão. Uma parcela significativa das chuvas é oriunda de convecção topográfica (Fisch 2003). A região da área do estudo é caracteristicamente representada como floresta ombrófila densa, genericamente chamada mata atlântica (Silva \& LeitãoFilho 1982, Veloso et al. 1991, Simonetti 2001).

A família Arecaceae é uma das famílias mais importantes na composição florística e estrutural desse trecho de Mata Atlântica, no município de Ubatuba. Além desta família, é característica a representatividade por famílias de dispersão universal, com um número de espécies significativas de Myrtaceae, Rubiaceae, Lauraceae e Melastomataceae (Silva \& Leitão-Filho 1982, Lacerda 2001).

As espécies Bactris setosa e B. hatschbachii, são palmeiras conhecidas localmente como tucum do brejo e tucum do cerro, respectivamente. Ambas apresentam excelentes fibras têxteis, que eram utilizadas como linha de pesca pelas populações caiçaras e as folhas, na confecção de cestarias (Medina 1959). As duas espécies apresentamse cobertas de espinhos (estipe, folhas e brácteas florais) e produzem frutos carnosos, que podem estar sendo fonte alimentícia para fauna frugívora.

\section{Procedimentos de campo}

As espécies foram amostradas na restinga correspondente à planície litorânea ( $0 \mathrm{~m}$ de altitude) e na encosta da Serra do Mar (100, 200, 400, 600 e 850 m). Em cada cota altitudinal, foi estendida uma linha de $200 \mathrm{~m}$ (transecto principal) acompanhando a superfície do terreno, ao longo da qual, foram sorteados dez pontos de onde partiram linhas secundárias com $50 \mathrm{~m}$ de comprimento (sub-transectos). Foram sorteados 25 pontos nos quais foram alocados os centros das parcelas circulares de $100 \mathrm{~m}^{2}$ (5,64 m de raio). Nestas parcelas circulares todos os indivíduos das duas palmeiras em estudo foram medidos quanto à altura do estipe até o ponto de abertura das folhas apicais. As espécies foram divididas em cinco classes de tamanho, conforme a maior altura do indivíduo de cada espécie, sendo: classe um (acaule); dois (1 a 50 cm); três (51 a 100 cm); quatro (101 a 200 cm); cinco (>200 


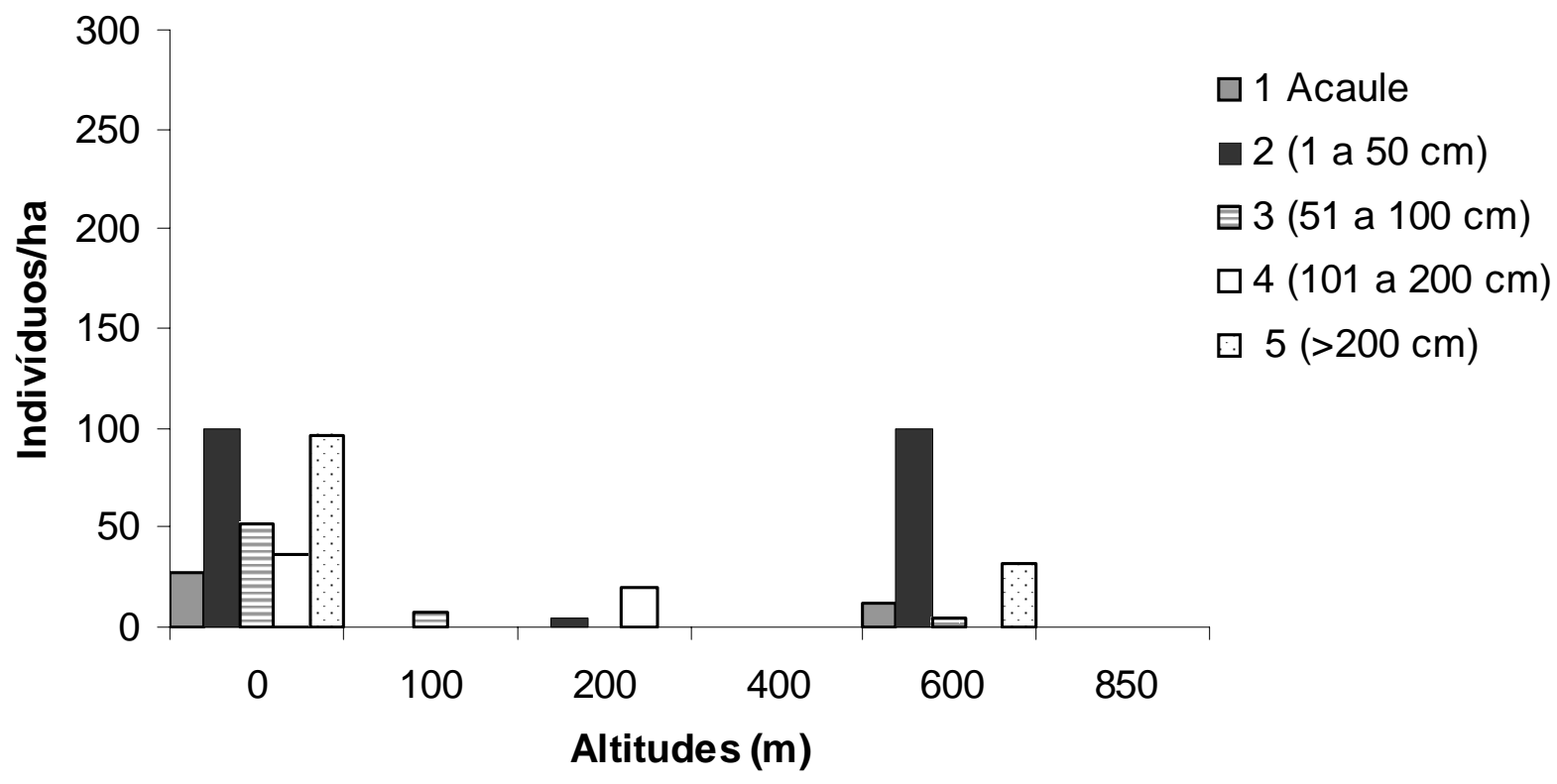

Figura 2: Distribuição de indivíduos/hectare de Bactris setosa Mart em classes de alturas em seis altitudes.

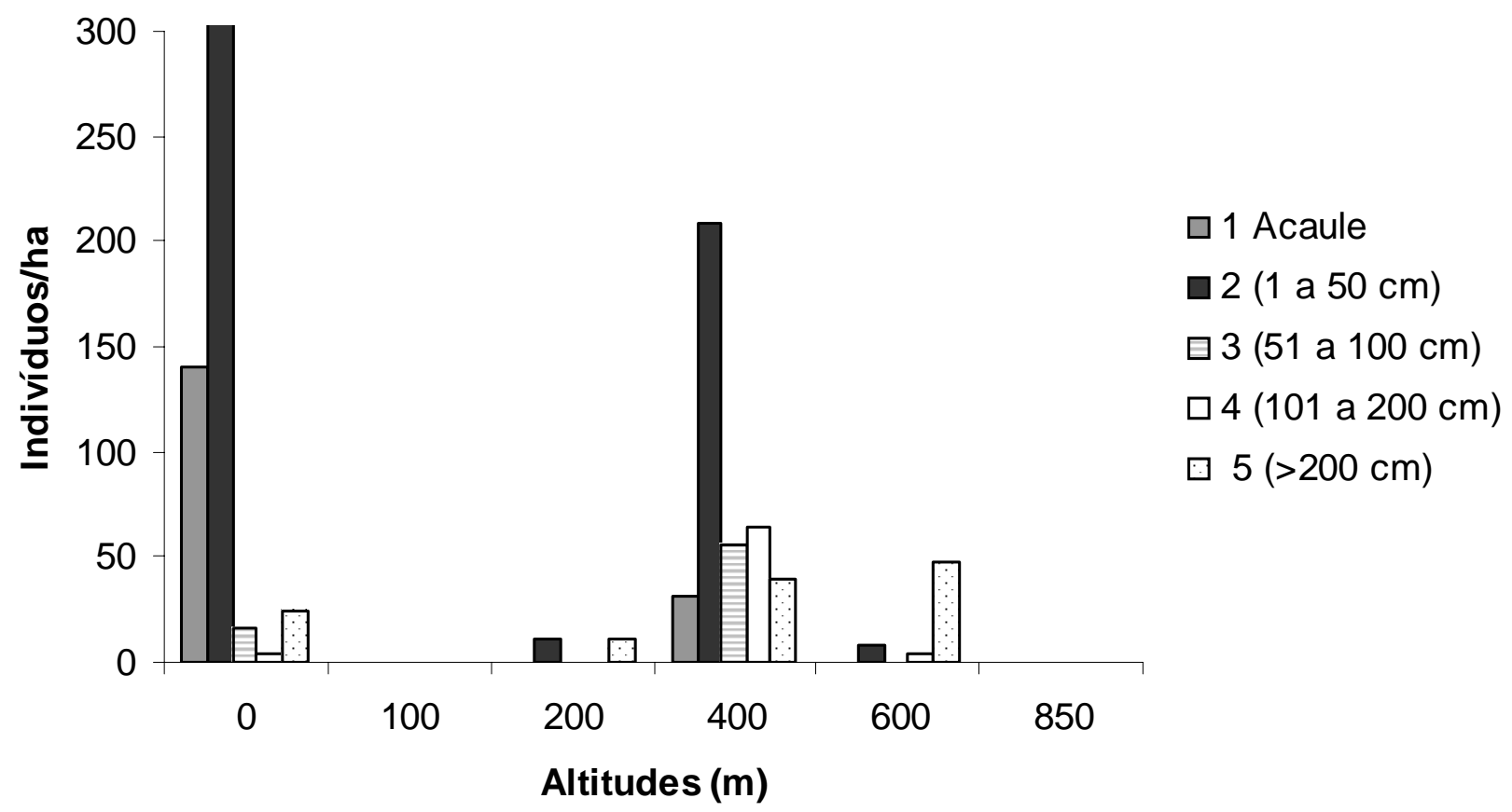

Figura 3: Distribuição de indivíduos/hectare de Bactris hatschbachii Noblick ex A. Hend, em classes de alturas em seis altitudes. 
Tabela 1: Resultados dos Índices de Morisita (Id) e qui-quadrado $\left(\div^{2}\right)$ das populações de Bactris hatschbachii $e$ B. setosa, amostradas em seis altitudes em um trecho de mata Atlântica/Ubatuba-SP.

\begin{tabular}{|c|c|c|c|c|c|c|c|c|c|c|c|c|c|c|}
\hline \multirow[b]{2}{*}{ Bactris hatschbachii } & \multicolumn{3}{|c|}{$0 \mathrm{~m}$} & \multicolumn{2}{|c|}{$100 \mathrm{~m}$} & \multicolumn{2}{|c|}{$200 \mathrm{~m}$} & \multicolumn{3}{|c|}{$400 \mathrm{~m}$} & \multicolumn{2}{|c|}{$600 \mathrm{~m}$} & \multicolumn{2}{|c|}{$850 \mathrm{~m}$} \\
\hline & Ind/hat+ & Id & $x^{2}$ & Id & $x^{2}$ & Id & $x^{2}$ & Ind/ha** & Id & $x^{2}$ & Id & $x^{2}$ & Id & $\mathrm{x}^{2}$ \\
\hline 1 (acaule) & $14 \pm 14,6$ & 2,58 & 77,72 & \begin{tabular}{|l|}
--- \\
\end{tabular} & $\begin{array}{l}--- \\
\end{array}$ & ---- & $-\overline{---}$ & $2,8 \pm 3,8$ & 1,78 & 29,46 & ---- & --- & \begin{tabular}{|l|}
--- \\
\end{tabular} & --- \\
\hline $2(1$ a $50 \mathrm{~cm})$ & $30,4 \pm 31,5$ & 1,94 & 93,56 & $\begin{array}{l}--- \\
-\end{array}$ & $\begin{array}{ll}--- \\
\end{array}$ & $*$ & $\begin{array}{ll}--- \\
\end{array}$ & $22,4 \pm 34,2$ & 4,8 & 214 & $*$ & $\begin{array}{ll}--- \\
\end{array}$ & ---- & $\begin{array}{ll}--- \\
\end{array}$ \\
\hline $3(51$ a $100 \mathrm{~cm})$ & $1,6 \pm 2,1$ & $*$ & --- & $\begin{array}{l}--- \\
--\end{array}$ & $\begin{array}{ll}--- \\
--\end{array}$ & $\begin{array}{ll}--- \\
--\end{array}$ & $-\cdots$ & $5,2 \pm 11$ & 8,97 & 119,6 & ---- & $\begin{array}{l}--- \\
--\end{array}$ & ---- & ---- \\
\hline $4(101$ a $200 \mathrm{~cm})$ & $0,4 \pm 1,3$ & $*$ & ---- & ---- & ---- & --- & $-\cdots$ & $6,8 \pm 10,8$ & 6,22 & 102,3 & $*$ & --- & $\begin{array}{ll}--- \\
-1\end{array}$ & --- \\
\hline $5(>200 \mathrm{~cm})$ & $1,6 \pm 3,9$ & 2,5 & 38 & $\begin{array}{ll}--- \\
\end{array}$ & $\begin{array}{ll}--- \\
\end{array}$ & $*$ & $\begin{array}{ll}--- \\
\end{array}$ & $4 \pm 6$ & 2,6 & 41,6 & $*$ & ---- & $\begin{array}{ll}--- \\
\end{array}$ & ---- \\
\hline Pop Total & $9,6 \pm 12,9$ & 1,73 & 110,1 & $\begin{array}{ll}--- \\
--\end{array}$ & ---- & $-\cdots$ & --- & $8,24 \pm 8,05$ & 5,07 & 431 & --- & --- & 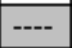 & --- \\
\hline Pop Tot(Indiha) & --- & 472 & +--- & --- & --- & 8 & --- & --- & 400 & ---- & 16 & --- & --- & --- \\
\hline Bactris setosa & Ind/hat+ & Id & $x^{2}$ & IdI & $x^{2}$ & Id & $x^{2}$ & Ind/hat+ & Id & $x^{2}$ & Id & $x^{2}$ & Id & $x^{2}$ \\
\hline 1 (acaule) & $2,4 \pm 3,4$ & 2,08 & 38,64 & ---- & ---- & ---- & ---- & ---- & $-\cdots$ & --- & $*$ & ---- & ---- & --- \\
\hline $2(1$ a $50 \mathrm{~cm})$ & $11,2 \pm 9,2$ & 2,1 & 76,5 & $\begin{array}{ll}--- \\
\end{array}$ & --- & $*$ & ---- & ---- & $-\cdots$ & $-\cdots$ & 4,41 & 105,8 & --- & --- \\
\hline $3(51$ a $100 \mathrm{~cm})$ & $5,6 \pm 6$ & 1,26 & 36,8 & $*$ & ---- & $*$ & ---- & ---- & ---- & $-\cdots$ & $*$ & \begin{tabular}{|l|}
---- \\
\end{tabular} & ---- & ---- \\
\hline $4(101$ a $200 \mathrm{~cm})$ & $4 \pm 4,6$ & 2,77 & 36,16 & --- & --- & --- & ---- & ---- & ---- & ---- & $\begin{array}{l}--- \\
\end{array}$ & $-\cdots$ & ---- & --- \\
\hline $5(>200 \mathrm{~cm})$ & $8,5 \pm 9,7$ & 2,35 & 55,05 & $-\cdots$ & ---- & $-\cdots$ & ---- & $-\cdots$ & $-\cdots$ & $-\cdots$ & $*$ & $-\cdots$ & ---- & $-\cdots$ \\
\hline Pop Total & $6,34 \pm 3,5$ & 1,79 & 84,83 & --- & --- & --- & --- & --- & --- & --- & 5,2 & 187,2 & \begin{tabular}{|l|}
--- \\
\end{tabular} & --- \\
\hline Pop Tot (Indiha) & \begin{tabular}{|l|}
--- \\
\end{tabular} & 312 & --- & 8 & ---- & 24 & ---- & ---- & --- & --- & 148 & --- & --- & --- \\
\hline \multirow{2}{*}{\multicolumn{14}{|c|}{ * Calculo impossibilitado pelo baixo número de indivíduos. }} & \\
\hline ** Médias e desvi & & $\mathrm{s}$ ind/h & a nas $\mathrm{cl}$ & lasses & e altu & & & & & & & & & \\
\hline
\end{tabular}

cm). As distribuições de freqüência das classes de tamanho das populações, para cada uma das cinco classes de alturas estabelecidas, foram analisadas através da construção de histogramas de freqüências.

A fim de verificar o padrão espacial das espécies, calculou-se o índice de Morisita (Id) para cada um dos estádios (Zar 1984). O padrão diferente do aleatório foi verificado pelo teste $\div^{2}$ (Krebs 1989) a partir da seguinte expressão: Id = n $\left(\mathrm{O} \mathrm{x}^{2}-\mathrm{N}\right) / \mathrm{N}(\mathrm{N}-1)$; onde: $\mathrm{n}=$ número de parcelas, $\mathrm{N}=$ número total de indivíduos presente em cada parcela e Ó $\mathrm{x}^{2}=$ somatório do quadrado do número de indivíduos por parcela. Neste estudo fez-se a análise do padrão espacial das populações em cada altitude separadamente (2.500 $\mathrm{m}^{2}$ distribuídos em vinte e cinco parcelas circulares de $100 \mathrm{~m}^{2}$ ), já que as espécies não ocorreram em todas altitudes.

\section{RESULTADOS}

\section{Classes de Alturas e Estrutura das Populações nas Altitudes}

As populações de ambas espécies apresentam maior concentração de indivíduos na classe dois (figuras 2 e 3). Este padrão estrutural deve-se ao fato das espécies perfilharem, somando-se os clones ("ramets”) aos indivíduos oriundos de sementes ("genets”). A distribuição corresponde para $B$. setosa a: 8\% classe um (acaule), 36\% classe dois (1 a 50 cm), 13\% classe três (51 a $100 \mathrm{~cm}), 13,5 \%$ classe quatro (101 a $200 \mathrm{~cm}$ ) e 29,5\% classe cinco (>200 cm, adultos), e para B. hatschbachii: $19 \%$ classe um, 58\% classe dois, $8 \%$ classe três, $8 \%$ e classe quatro, 7\%. Porém, na classe de maior tamanho verificou-se que $B$. setosa apresentou uma freqüência maior de indivíduos que $B$. hatschbachii, indicando para esta última uma população mais jovem. A despeito de apresentar-se mais madura, a população de $B$. setosa foi menos numerosa que $B$. hatschbachii.

Em relação à distribuição altitudinal (Tab. 1, figuras 2 e 3), as duas espécies não ocorreram nos $850 \mathrm{~m}$, ponto mais elevado nas amostragens. Ao nível do mar, as populações de $B$. setosa e $B$. hatschbachii apresentaramse mais densas e distribuídas de forma homogênea dentro das classes de alturas. A $100 \mathrm{~m}$ de altitude observou-se somente oito ind/ha de B. setosa. A $200 \mathrm{~m}$ ambas ocorreram em baixa densidade; a 400 m de altitude observou-se a grande concentração da espécie $B$. hatschbachii e ausência de $B$. setosa, enquanto a $600 \mathrm{~m}$ ocorreu praticamente o inverso, presença de $B$. setosa e poucos indivíduos de B.hatschbachii.

Na encosta, a $400 \mathrm{~m}$ a população de $B$. hatschbachii apresentou densidade próxima à observada na restinga (Tab. 1, fig. 3), porém com maior número de indivíduos nas classes 3, 4 e 5 (jovens e adultos). Nos 600 m (fig. 2), ponto mais populoso de $B$. setosa na encosta, a densidade observada para esta espécie foi menos que a metade da alcançada na 
restinga. Apesar dos indivíduos da classe 2 apresentarem o mesmo valor observado na restinga, as demais classes apresentaram pequeno número de indivíduos e a classe 4 não ocorreu nesta altitude.

\section{Padrão Espacial das Populações}

Na restinga, a população de $B$. hatschbachii apresentou um padrão espacial agregado assim como, nas classes de altura o padrão espacial foi também agregado. Aos $400 \mathrm{~m}$ de altitude, o padrão indicado foi também agregado, da mesma forma que para todas as classes de desenvolvimento, apresentando porém um índice de agregação menor na classe inicial (Tabela 1).

A população de $B$. setosa na restinga apresentou um padrão espacial agregado. Esse mesmo padrão foi verificado para todas as classes de alturas. Nos 600 m de altitude o padrão espacial foi agregado para a população total. Nessa altitude a única classe de altura em que foi possível calcular separadamente o índice de Morisita, foi a classe 2 (1 a 50 $\mathrm{cm}$ ) a qual apresentou um índice significativo de agregação. Nas outras altitudes investigadas o número de indivíduos foi insuficiente para o cálculo.

\section{DISCUSSÃO}

A ausência das duas espécies nos $850 \mathrm{~m}$ de altitude, a baixa ocorrência de indivíduos nos 100 e 200 m, e a concentração na restinga, nos 400 m (B. hatschibachii) e nos $600 \mathrm{~m}$ (B. setosa), pode estar relacionada a eventos estocásticos, como agentes dispersores, nichos adequados para germinação e estabelecimento das plântulas. Monteiro (2004) destaca que 100 e 200 m foram cotas com maiores variações de declividades e solo menos úmido, e aos $850 \mathrm{~m}$ de altitude o solo também foi pouco úmido e o dossel menos denso. Correlações com diversos fatores do meio indicaram que estas palmeiras são exigentes e dependentes de fatores edáficos locais, como fertilidade e umidade do solo (Monteiro 2004).

De acordo com a revisão de Moore (1973), as palmeiras ocupam diversos habitat em diferentes altitudes, sendo porém ausentes em ambientes verdadeiramente xéricos e apresentam uma forte predileção por locais úmidos, aonde chegam ser dominantes, como observado pelas manchas populacionais formadas pelas espécies estudadas. Em levantamento realizado em outra área da Serra do Mar na mesma região, Lacerda (2001) também observou distribuição restrita a determinadas altitudes (0 e $600 \mathrm{~m}$ ) para B. setosa.

Em geral as palmeiras apresentam susceptibilidade ao frio (Larcher \& Winter 1981), que pode provocar danos no meristema apical e como escape algumas espécies perfilham (Richards 1996). No entanto, a limitação altitudinal apresentada pelas palmeiras na encosta estudada não parece motivada pela diferença da temperatura ao longo do gradiente, mas pela ação desta em conjunto com fatores edáficos.
Em gradientes altitudinais, a estrutura e principalmente a distribuição espacial das espécies em geral, que normalmente diminui com a elevação, está relacionada com a precipitação e diminuição da temperatura ocasionada pela presença constante de neblina (Gentry 1988, Lieberman et al. 1996, Richards 1996). Estas mudanças ambientais fazem com que algumas espécies tendem a estabelecer-se e agregarse em determinadas altitudes, fato constatado por Santos et al. (1998) e Lacerda (2001) em um trecho de mata Atlântica, onde o maior número de espécie ocorreu entre os 300 e 600 m de altitude, e a abrupta diminuição das palmeiras nos 1000 m, sugerindo um padrão de ocorrência para esta família em gradientes altitudinais.

O padrão de distribuição agregado para as populações e para praticamente todas as classes de altura de $B$. hatschbachii e B. setosa, difere do padrão agregado nos estádios iniciais, e aleatório nos estádios seguintes apresentado pelas palmeiras: Manicaria martiana (Ribeiro 1991), Euterpe edulis (Reis et al. 1996, Fisch 1999, Silva-Matos et al. 1999) e Syagrus romanzoffiana (Bernacci 2001). Estas palmeiras, no entanto, apresentam estipe único e ocupam o dossel florestal, enquanto que $B$. hatschbachii e $B$. setosa formam touceiras e são típicas de subosque. Segundo De Steven \& Putz (1985), palmeiras de subosque são mais susceptíveis a mortalidade que as do dossel, devido a danos no meristema apical causados pela fauna herbívora e queda de árvores. Neste sentido a forma de vida multicaulinar das espécies estudadas pode ser uma estratégia de sobrevivência à situação ambiental em que estão inseridas. Assim, para compreensão do padrão encontrado sugere-se estudos mais detalhados sobre as espécies.

Entretanto, não se pode afirmar que as espécies apresentam um padrão estrutural e espacial estável. Devese considerar, como comentou Santos (1991), além das relações vegetacionais como um todo e as alterações ocorridas ao longo do tempo. Eventos reprodutivos, quedas de árvores, assim como as relações intra e interespecificas podem em determinado momento causar modificações temporárias na estrutura e padrão espacial das espécies.

Neste estudo ambas espécies encontraram-se bem estabelecidas e em algumas situações dividindo o mesmo espaço, como o observado na restinga. Esse fato indica que não está ocorrendo competição entre as mesmas, pelo menos nestes locais onde ocorre abundância hídrica. Em altitudes onde ocorreu representante de apenas uma espécie, o agregamento observado mostra indícios de que as espécies passaram por processos adaptativos que podem ter determinado ou não o sucesso do estabelecimento da população em certos locais. Esta situação pode ter sido provocada pela estratégia reprodutiva apresentada pelas espécies, reprodução sexuada via sementes e assexuada via entouceiramento. Neste primeiro caso a fauna local tem papel importante, e uma vez estabelecida, a touceira garante a perpetuação no ambiente por um período maior. 
De uma forma geral, pôde-se comprovar que as palmeiras estudadas, componentes do subosque da floresta ombrófila densa atlântica, possuem distribuição restrita a locais que favoreceram seu estabelecimento.

\section{AGRADECIMENTOS}

Os autores agradecem ao Programa Biota/FAPESP (01/06023-5) pelo apoio financeiro, ao Prof. Dr. Eduardo Pereira Cabral Gomes (UNITAU) pela colaboração e sugestões prestadas, ao Chefe da Estação Experimental de Ubatuba, Engo. Agr. Gentil Godoy Filho pelas acomodações cedidas durante o desenvolvimento deste trabalho e ao senhor Rosendo “Jones" Messiano pela colaboração nos trabalhos de campo.

\section{REFERÊNCIAS BIBLIOGRÁFICAS}

BERNACCI, L.C. 2001. Aspectos da demografia da palmeira nativa Siagrus romanzoffiana (Cham.) Glasman, jerivá, como subsídio ao seu manejo. Tese de doutorado. Campinas, SP.

DE STEVEN, D. \& PUTZ, F.E. 1985. Mortality rates of some rain Forest palms in Panama. Principes 29(4): 162-165.

EDWARD, P.J. \& GRUBB, P.J. 1982. Studies of mineral cycling in a montane rain forest in New Guinea. Soil characteristics and the division of mineral elements between the vegetation and soil. Journal of Ecology 70: 649-666.

EWEL, J.J. \& BIGELOW, S.W. 1996. Plant life-forms and tropical ecosystem functioning. In: ORIANS, G.H. ; DIRZO, R. ; CUSHMAN, J.H. (eds). Biodiversity and ecosystem processes in Tropical Forests. Berlin, Springer-Verlag, $229 \mathrm{p}$.

FISCH, G. 2003. Distribuição da comunidade de palmeiras no gradiente altitudinal da Floresta Atlântica na região nordeste do estado de São Paulo. Resumo In IV Simpósio e IV Reunião de Avaliação do Programa Biota/Fapesp. Águas de Lindóia.

FISCH, S.T.V. 1999. Dinâmica de Euterpe edulis Mart. na Floresta Ombrófila Densa Atlântica em Pindamonhangaba - SP. Tese de Doutorado. São Paulo, Instituto de Biociências, Universidade de São Paulo, 126 p.

GENTRY, A.H. 1988. Changes in plant community diversity and Floristic composition on environmental and geographical gradients. Annals of Missouri Botanical Garden, 75: 1- 34 .

GRUBB, P.J. 1977. Control of Forest growth and distribution on wet tropical mountains: With special reference to mineral nutrition. Annual Review of Ecology and Systematics 8: 83-107.

KENT, M. \& COKER, P. 1992. Vegetation description and analysis - A pratical approach. Belhaven Press, London.

KREBS, C. J. 1989. Ecological Metodology. New York, Library of Congress. 659 p.
LACERDA, M. S. 2001. Composição florística e estrutura da comunidade arbórea num gradiente altitudinal da Mata Atlântica. Tese de Doutorado. Campinas, SP.

LARCHER, W. \& WINTER, A. 1981. Frost susceptibility of palm: Experimental data and their interpretation. Principes, 25(4): 143-152.

LIBERMAN, D., LIBERMAN, M., PERALTA, R. \& HARTSHORN, G. J.S. 1996. Tropical forest structure and composition on large scale altitudinal gradient in Costa Rica. Journal of Ecology 84: 137-152.

MEDINA, J. C. 1959. Plantas fibrosas da flora mundial. Instituto Agronômico de Campinas. Indústria Gráfica Siqueira S/A, p. 140-143.

MONTEIRO, E.A. 2004. Caracteres morfológicos e influência ambiental nas populações de Bactris hatschbachii Noblick ex A. Hend e Bactris setosa Mart (Arecaceae), no gradiente altitudinal em um trecho de mata Atlântica/Ubatuba-SP. Dissertação de Mestrado. Universidade de Taubaté, SP.

MOORE Jr, H.E. 1973 Palms in the tropical Forest Ecosystems of África and South America. In: MEGGERS, B.J.; AYENSER, E.S.; DUCKWORTH, W.D. (eds). Tropical Forest Ecosystems in Africa and South America: A Comparative Review. Washington, Smithsoniam Ist. Press, p. 63-88.

REIS, A.; KAGEYAMA, P.Y.; REIS, M.S. \& FANTINI, A. 1996. Demografia de Euterpe edulis Martius (Arecaceae) em uma floresta ombrófila densa Montana, em Blumenau (SC). Sellowia, 45-48: 13-45.

RIBEIRO, A. S. 1991. Estrutura e dinâmica de uma população de bussu Manicaria martiana Burret (Arecaceae), em floresta úmida de terra firme na Amazônia Central, Manaus, Brasil. Dissertação de Mestado. Universidade do Amazonas. Manaus - Amazonas.

RICHARDS, P.W. 1996. The Tropical Rain Forest. 2 ed. Cambridge, Cambridge University Press, 575 p.

SANTOS, F.A.M. 1991. Padrão espacial de jovens em relação a adultos de espécie arbóreas de cerrado que ocorrem no estado de São Paulo. Tese de Doutorado. Campinas, Universidade Estadual de Campinas.

SANTOS, F.A.M. 1998. Structure and dynamics of tree species of the Atlantic Forest. An. Acad. Bras. Ci., Rio de Janeiro. 70 (4): 873-880.

SILVA, A.F. \& LEITÃO FILHO, H.F. 1982. Composição florística e estrutura de um trecho de mata atlântica de encosta no município de Ubatuba (São Paulo, Brasil). Revista brasileira de botânica, 5:43-52.

SIVA-MATOS, D.M.; FRECKLETON, R.P. \&WATKINSON, A.R. 1999. The role of density dependence in population dynamics of tropical palm. Ecology 80(8): 2635-2650.

SIMONETTI, C. 2001. As relações entre o relevo, os solos e a Floresta Atlântica na Serra do Mar (Bacia do rio Itamambuca, Ubatuba, SP). Tese de doutorado. Universidade de São Paulo, SP. 
SOUZA, A.F.; MARTINS, F.R. \& BERNACCI, L.C. 2003.

Clonal growth and reproductive strategies of the understory tropical palm Geonoma brevispatha: an ontogenetic approach. Can. J. Bot. 81: 101-112.

SOUZA, A.F.; MARTINS, F.R. \& SILVA-MATOS, D.M. 2000. Detecting ontogenetic stages of the palm Attalea humilis in fragments of the Brazilian Atlantic forest. Can. J. Bot. 78: $1227-1237$.

SVENNING, J.-C. 2001. Evironmental heterogeneity, recruitment limitation and the mesoscale distribution of palms in a tropical montane rain forest (Maquipucuna, Ecuador). Journal of Tropical Ecology 17: 97-113.

VELOSO, H. P.; RANGEL FILHO, A. L. R.; LIMA, J. C. A. 1991. Classificação da vegetação brasileira, adaptada a um sistema universal. IBGE, Rio de Janeiro.

ZAR, J.H. 1984. Biostatistical analysis. Prentice-Hall Internacional Editions, New Jersey.

Título: Estrutura e padrão espacial das populações de Bactris setosa Mart e B. hatschbachii Noblick ex A. Hend (Arecaceae) em um gradiente altitudinal, Ubatuba (SP).

Autores: Evoni Antunes Monteiro, Simey Thury Vieira Fisch

Biota Neotropica, Vol. 5 ( número 2): 2005

http://www.biotaneotropica.org.br/v5n2/pt/ abstract?article+BN00505022005

Recebido em 20/04/04 - Revisado em 04/03/05.

Publicado em 01/07/2005.

ISSN 1676-0603

http://www.biotaneotropica.org.br 